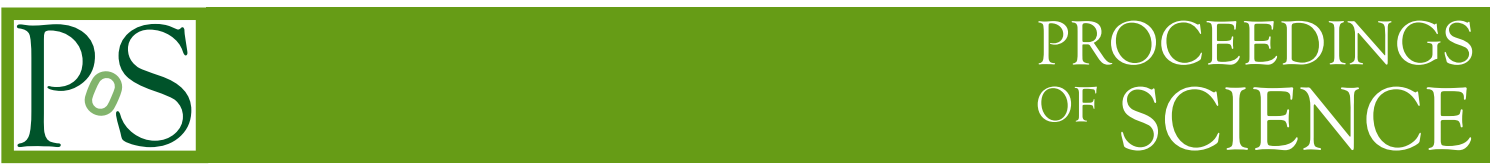

\title{
Measurement of the proton scalar polarizabilities at MAMI
}

\author{
Edoardo Mornacchi* on the behalf of the A2 Collaboration \\ Johannes Gutenberg - Universität Mainz \\ E-mail: emornacc@uni-mainz.de
}

\begin{abstract}
The electric $\left(\alpha_{\mathrm{E} 1}\right)$ and magnetic $\left(\beta_{\mathrm{M} 1}\right)$ scalar polarizabilities are fundamental properties related to the internal structure of the nucleon. They play a crucial role not only in our understanding of the nucleon, but also in other areas, such as atomic physics. In the past, the values of $\alpha_{\mathrm{E} 1}$ and $\beta_{\mathrm{M} 1}$ were determined from the unpolarized differential cross-section of Compton scattering $\gamma p \rightarrow \gamma p$. The measurement of the beam asymmetry $\Sigma_{3}$ provides an alternative approach to the extraction of the scalar polarizabilities with different sensitivity and systematics compared to the unpolarized cross-section. This asymmetry was measured for the first time below the pion photoproduction threshold by the A2 Collaboration with the Crystal Ball/TAPS experiment at MAMI (Mainz, Germany). A linearly polarized photon beam impinged on a liquid hydrogen target and the scattered photons were detected with the Crystal Ball/TAPS setup, providing almost $4 \pi$ coverage. A new high precision measurement of both unpolarized cross-section and beam asymmetry $\Sigma_{3}$ will be performed in the near future and polarizabilities $\alpha_{\mathrm{E} 1}$ and $\beta_{\mathrm{M} 1}$ will be extracted with unprecedented precision.
\end{abstract}

XVII International Conference on Hadron Spectroscopy and Structure

25-29 September, 2017

University of Salamanca, Salamanca, Spain

\footnotetext{
*Speaker.
} 


\section{Introduction to scalar polarizabilities}

The scalar dipole polarizabilities $\alpha_{\mathrm{E} 1}$ and $\beta_{\mathrm{M} 1}$ describe the response of a nucleon to an applied static electric and magnetic field, respectively. They are not only of great importance for nuclear and atomic physics, but they limit the precision in many other areas of physics. They contribute at the second order in the low-energy expansion of Compton scattering on proton $(\gamma p \rightarrow \gamma p)$ [1], and can be accessed by measuring at sufficiently low energy $\left(\omega<m_{\pi}\right)$ the differential unpolarized cross section and the asymmetry $\Sigma_{3}$, available with a linearly polarized beam [2]. The current values of the proton scalar polarizabilities quoted by the Particle Data Group (PDG) [3] are $\alpha_{\mathrm{E} 1}=$ $(11.2 \pm 0.4) \times 10^{-4} \mathrm{fm}^{-3}$ and $\beta_{\mathrm{M} 1}=(11.2 \pm 0.4) \times 10^{-4} \mathrm{fm}^{-3}$. So far, they have been determined only from the unpolarized cross section measured in different Compton scattering experiments, and by far the largest data set was obtained at MAMI [4].

\section{Experiment}

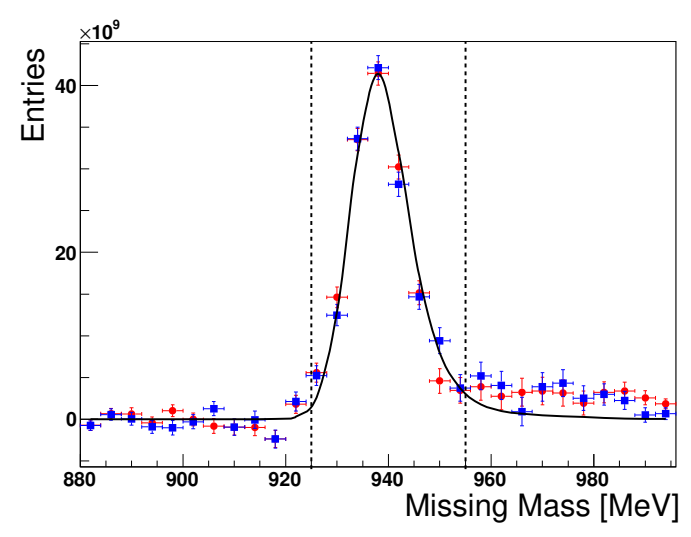

(a)

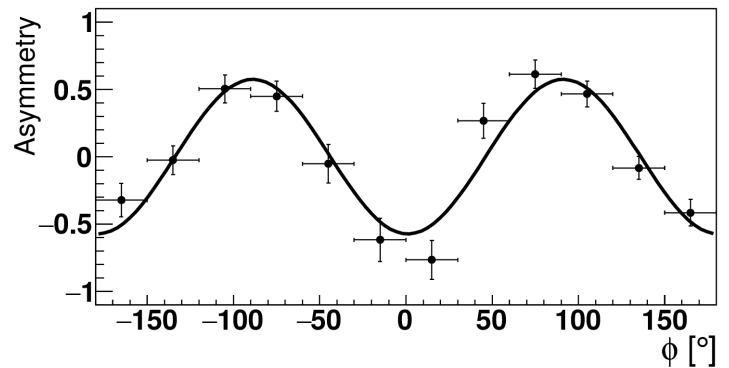

(b)

Figure 1: (a) Missing mass spectra for $E_{\gamma}=79-98 \mathrm{MeV}$ and $30^{\circ}<\theta<155^{\circ}$ for the two polarization planes (parallel and perpendicular, red circles and blue squares respectively) compared with the Monte Carlo simulated distribution for Compton scattering events (solid black line). (b) $\phi$-distribution for $E_{\gamma}=119-$ $139 \mathrm{MeV}$ and $100^{\circ}<\theta<120^{\circ}$ obtained from eq. 3.2. Figure taken from [7].

An experiment was performed at the MAMI facility in Mainz [5], using a beam of linearly polarized tagged photons, obtained via coherent bremsstrahlung on a thin diamond radiator. The photons impinged on a $10 \mathrm{~cm}$ liquid hydrogen target. The reaction products were then detected using the Crystal Ball/TAPS apparatus of the A2 Collaboration, covering nearly $97 \%$ of the full solid angle. Further information about the A2 setup can be found in [6].

The measurements were performed with two different orientations of the photon polarization vector with a relative shift of $90^{\circ}$ in respect to each other. To select Compton events, a single neutral particle in the Crystal Ball was required, since the outgoing proton is stopped inside the target at the energy range relevant for our analysis $\left(E_{\gamma}=79-139 \mathrm{MeV}\right)$. To reduce the random background in the Crystal Ball, a time-coincidence between the scattered photon and the tagger hit was required and a random background subtraction was performed. Furthermore, to remove background events 
originating from the target cell, an empty target contribution was also performed. Figure 1a shows the corresponding missing mass spectra. The good agreement between both the data sets and the Monte Carlo spectrum indicates low background contamination as well as absence of significant systematic effects. The two dotted lines in Fig. 1a indicate the cut on the missing mass applied for all the three energy bins. After these cuts, a final data sample consisting of more than $2 \times 10^{5}$ Compton events was used for the extraction of the beam asymmetry $\Sigma_{3}$.

\section{Beam asymmetry $\Sigma_{3}$}

The use of linearly polarized photons with an unpolarized target introduces an azimuthal dependence to the cross section that, defining $\phi$ as the angle between one of the polarization planes and the scattering plane, can be written as:

$$
\sigma_{\mathrm{pol}}=\sigma_{\mathrm{unpol}}\left(1+\delta_{l} \Sigma_{3} \cos (2 \phi)\right)
$$

where $\sigma_{\text {unpol }}$ is the unpolarized cross section, $\delta_{l}$ is the degree of linear polarization of the beam, $\Sigma_{3}$ defines the amplitude of the $\cos (2 \phi)$ modulation. To account for different polarization values for the two sets with different orientation of the diamond, the number of Compton scattering events was weighted with the corresponding linear polarization values in the expression for $\Sigma_{3}$ derived from eq. 3.1

$$
\Sigma_{3} \cos \left(2\left(\phi+\phi_{0}\right)\right)=\frac{\sigma_{\|}-\sigma_{\perp}}{\delta_{\perp} \sigma_{\|}-\delta_{\|} \sigma_{\perp}}=\frac{N_{\|}-N_{\perp}}{\delta_{\perp} N_{\|}-\delta_{\|} N_{\perp}},
$$

where $\delta_{\|(\perp)}$ and $\mathrm{N}_{\|(\perp)}$ represent respectively the degree of linear polarization and the event rates for the two settings, and $\phi_{0}$ corresponds to the possible deviation of the polarization planes from the expected position.

The beam asymmetry $\Sigma_{3}$ was extracted by fitting the $\phi$ distributions (see Fig. 1b) and equating to $\sin (\Delta \phi) / \Delta \phi \Sigma_{3} \cos \left(2\left(\phi+\phi_{0}\right)\right)$ in eq. 3.2 (where the prefactor $\sin (\Delta \phi) / \Delta \phi$ accounts for the width of the bins in $\phi$ ). The results shown in Fig. 2 are in good agreement with the theoretical calculations within BChPT [8], HBChPT [9] and DR [10]. Instead of fixing the polarizability values, they can be fitted directly to the $\Sigma_{3}$ data. The fit was performed using only the new data on $\Sigma_{3}$ presented

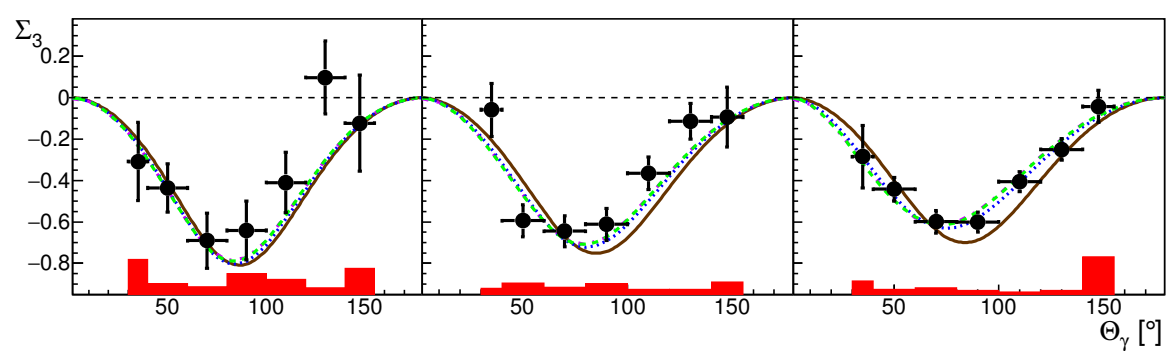

Figure 2: Beam asymmetry $\Sigma_{3}$ for the three energy ranges (left: $E_{\gamma}=79-98 \mathrm{MeV}$, middle: $E_{\gamma}=98-$ $119 \mathrm{MeV}$, right: $E_{\gamma}=119-139 \mathrm{MeV}$ ). The error represent statistical errors only, the systematic errors are indicated by the red bars. Brown solid curve: Born contribution, green dashed curve: BChPT calculation [8], blue dotted curve: HBChPT calculation [9], magenta dash-dotted curve: DR calculation [10], all fixing $\alpha_{\mathrm{E} 1}=10.65 \times 10^{-4} \mathrm{fm}^{-3}$ and $\beta_{\mathrm{M} 1}=3.15 \times 10^{-4} \mathrm{fm}^{-3}[7]$. 


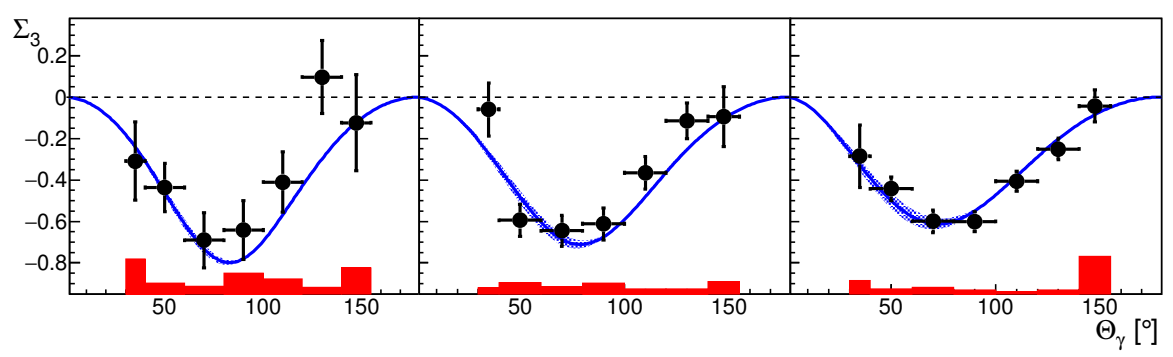

Figure 3: Results of the fit within BChPT framework [8] obtained by averaging over angle and energy. Shaded blue bands represent the error in the value of $\beta_{M 1}$ coming from the fit. Energy bins and notation for the data as in Fig. 2 [7].

in this work [7] within the BChPT [8] and HBChPT [9] frameworks and it was constrained by Baldin sum rule. Figure 3 shows the BChPT result (very similar plot is obtained for the HBChPT fit). The two fits return respectively $\beta_{\mathrm{M} 1}=2.8_{-2.1}^{+2.3} \times 10^{-4} \mathrm{fm}^{3}\left(\chi^{2} / n d f=19.2 / 20\right)$ and $\beta_{\mathrm{M} 1}=$ $3.7_{-2.3}^{+2.5} \times 10^{-4} \mathrm{fm}^{3}\left(\chi^{2} / \mathrm{nd} f=17.1 / 20\right)$. A new unprecedented high-precision measurement is planned in the near future at MAMI with significantly higher statistics.

The author acknowledges support from the Collaborative Research Center (CRC) 1044.

\section{References}

[1] D. Babusci, G. Giordano, A.I. L'vov, G. Matone, A.M. Nathan, Low-Energy Compton Scattering of Polarized Photons on Polarized Nucleons, Phys. Rev. C 58, 1013 (1998).

[2] N. Krupina, V. Pascalutsa, Separation of Proton Polarizabilities with the Beam Asymmetry of Compton Scattering, Phys. Rev. Lett. 110, 262001 (2013).

[3] C. Patrignani, et al. (Particle Data Group), Review of Particle Physics, Chin. Phys. C 40, 100001 (2016) and 2017 update.

[4] V. Olmos de Leon, et al., Low-energy Compton scattering and the polarizabilities of the proton, Eur. Phys. J. A 10, 207 (2001).

[5] K.-H. Kaiser, et al., The 1.5 GeV harmonic double-sided microtron at Mainz University, Nucl. Instrum. Methods 593, 159 (2008).

[6] A. Neiser (A2 Collaboration), Current Status and Performance of the Crystal Ball and TAPS Calorimeter, J. Phys. Conf. Ser. 587, 012041 (2015).

[7] V. Sokhoyan, E.J. Downie, E. Mornacchi, J.A. McGovern, N. Krupina, et al. (A2 Collaboration), Determination of the scalar polarizabilities of the proton using beam asymmetry $\Sigma_{3}$ in Compton scattering, Eur. Phys. J. A 53, 14 (2017).

[8] V. Lensky, V. Pascalutsa, Predictive powers of chiral perturbation theory in Compton scattering off protons, Eur. Phys. J. C 65, 195 (2010).

[9] J.A. McGovern, D.R. Philips, H.W. Grießhammer, Compton scattering from the proton in an effective field theory with explicit Delta degrees of freedom, Eur. Phys. J. A 49, 12 (2013).

[10] B. Pasquini, D. Drechsel, and M. Vanderhaeghen, Proton spin polarizabilities from polarized Compton scattering, Phys. Rev. C 76, 015203 (2007). 